\title{
Functionalized Asymmetric Poly (Lactic Acid)/Gelatin Composite Membrane for Guided Periodontal Tissue Regeneration
}

\author{
Shuangying Gu, Baoxiang Tian, Weicong Chen, Yue Zhou* \\ School of Biomedical Engineering and Med-X Research Institution, Shanghai Jiao Tong University, Shanghai, China \\ Email: *yzhou2009@sjtu.edu.cn
}

How to cite this paper: Gu, S.Y., Tian, B.X., Chen, W.C. and Zhou, Y. (2017) Functionalized Asymmetric Poly (Lactic Acid)/Gelatin Composite Membrane for Guided Periodontal Tissue Regeneration. Journal of Biomaterials and Nanobiotechnology, 8, 229-244. https://doi.org/10.4236/jbnb.2017.84016

Received: August 4, 2017

Accepted: September 22, 2017

Published: September 25, 2017

Copyright $\odot 2017$ by author and Scientific Research Publishing Inc. This work is licensed under the Creative Commons Attribution International License (CC BY 4.0).

http://creativecommons.org/licenses/by/4.0/

\begin{abstract}
Aim: Periodontitis is caused by chronic gingival inflammation and affects a large population in the world. Although guided tissue regeneration (GTR) therapy has been proven to be an effective treatment, the deficiency in the symmetrical design of all the GTR membrane in the market leaves large space for improvement. Therefore, we designed a novel asymmetrical bi-layer PLA/gelatin composite membrane for treating periodontitis. Methods: The PLA side was fabricated by electrospinning with metronidazole (MNA) pre-mixed with the PLA solution. The gelatin side containing bioglass (BG) $45 S 5$ was fabricated with freeze-drying process and cross-linked with PLA membrane. The bio-compatibility of the membrane was evaluated in vitro using NIH3T3 cells. The releasing of MNA was measured by spectrophotometer. The bioactivity of the membrane was evaluated by hydroxyapatite (HA) deposit and determined by FTIR spectrometer. The ionic concentration of $\mathrm{Ca}^{2+}$ and $\mathrm{SiO}_{4}^{2-}$ was measured by ICPOES. The expression of the osteogenesis makers was determined by qRT-PCR. Results: The bi-layer PLA/gelatin composite membrane is biocompatible and bioactive. The releasing of MNA can rapidly reach the anti-bacterial effective concentration. Interestingly, the incorporation of MNA modulated the degradation rate of PLA scaffold to meet the requirement of tissue regeneration. Meanwhile, the embedding of the BG powder in the gelatin porous layer provided a favorable $\mathrm{Ca}^{2+}$ and $\mathrm{SiO}_{4}^{2-}$ ion environment for the regeneration of the alveolar bone tissue. Conclusions: Taken together, this bi-layer GTR membrane is closer to the physiological structure of the periodontal. The addition of MNA and BG makes it more powerful in treating periodontitis. Moreover, this research provides an example of biomimetic design in fabricating biomaterial for clinical applications.
\end{abstract}




\section{Keywords}

Periodontitis, Guided Tissue Regeneration, Electrospin, Composite Membrane, PLA, Gelatin

\section{Introduction}

Peridontitis is a chronic gingival inflammation that affects a worldwide population up to $90 \%$ [1]. Most people will not pay attention to this disease when initial signs of gingivitis appear, such as bleeding while biting on food or sensitivity to temperature. However, as a matter of fact, with the progression of this disease, both tooth and the surrounding gingival tissue get involved in the damage process, and become irreversible. Peridontitis even involves with serious complications including diabetes [2] [3] and also is an independent risk factor of coronary heart disease [4].

Conventional periodontal treatment has been focused on periodontal guided tissue regeneration (GTR) therapy [5] since it is the most effective therapy up to date. The GTR membrane is basically a tissue engineering scaffold inserted between the periodontal ligament and alveolar bone. Since the reparative potential of periodontium is extremely limited in adult teeth, the GTR is required to induce the regenerative ability of the damaged tissue and the regeneration has to be in the form of both hard tissue (alveolar bone and cementum) and soft tissue (periodontal ligament). Therefore, to seek better GTR membranes to treat peridontitis, one has to bear with the idea that: 1) Simplicity in fabrication is desirable and more practical to be translated into clinical application; 2) The membrane is asymmetrically designed to mimic the real tissue organization; 3) The membrane is preferred to be bio-degradable but the degradation rate has to be fine-tuned to sustain enough mechanical strength to provide enough support for the cells to grow in the scaffold. To test these ideas, we designed and fabricated an asymmetrical PLA/gelatin bi-layer composite membrane for treating periodontitis.

Poly (lactic acid) (PLA) is a synthetic material that has been widely used to fabricate clinic sutures and bondages, etc. Bottino et al. [6] presented a smartly designed and functionally graded structure using PLA electros pinning membrane sandwich to tailor the degradation time and proper tensile strength. PLA is safe, while its degradation rate is relatively slow (more than 6 months) depending on the average molecular weight. However, according to our previous research, if mixed with other chemical, it was possible to adjust the degradation time to desired point by adjusting the proportion of the components in the mixture [7].

Clinical experience has reflected that bacterial infection is not only involved in the etiology of periodontitis but also becomes an obstacle for tissue regeneration [8]. In addition to the materials used for designing an ideal composite membrane for GTR, another aspect is also need to consider is to minimize the bacterial 
infection in the whole treatment process. 2-methyl-5-nitroimidazole-1-ethanol (MNA) is an effective drug for treating anaerobic bacteria infection in mouth and intestinal tract. Since the degradation time of PLA is adjustable by mixing with other agents, we attempted to mix MNA while fabricating electrospinning nanofibrous PLA to decrease the PLA degradation rate and control-releasing MNA.

Gelatin is derived from collagen and is composed of peptides and proteins of various molecular weight, commonly used in food, cosmetics and pharmaceuticals. Gelatin can be fabricated to mimic the natural extracellular matrix and showed a very promising advantage in soft tissue regeneration [9]. In designing this composite membrane, in order to both enhance the mechanical strength and increase the biocompatibility to gingiva, gelatin was considered as the basal of the other side of the bi-layer composite membrane.

Bioglass (BG) is a well-known bioactive material. Since its discovery by L. Hench, BG has been applied as bone fillers, bone repair materials and adjuvants in bone grafts due to their enhanced biocompatibility, osteoconductive and osteoinductive properties (Peter et al., 2009, 2010). BG can form a carbonated apatite layer between hard and soft tissues. Bioactive glass ceramics have been reported to influence osteoblastic cell differentiation with an increase in the level of differentiation markers like alkaline phosphatase (ALP), osteocalcin and osteopontin [10]. Therefore, BG was also considered to be an active component while fabricating our bi-layer GTR membrane.

In summary, in this paper, we report a smartly designed asymmetrical PLA/gelatin composite membrane for treating periodontitis. It was "functionalized" since the membrane contains an anti-inflammation drug, metronidazole (MNA), on one-side and bioglass 45S5 to induce osteogenesis on the other side.

\section{Materials and Methods}

\subsection{Chemicals and Cell Lines}

Poly (lactic acid) (PLA) and gelatin was supplied by Jinan Daigang Co. (Jinan, China). 2-methyl-5-nitroimidazole-1-ethanol (Metronidazole, MNA) was acquired from Aladdin Co. (Shanghai, China). Bioglass 45S5 (with a composition mole percentage of $\mathrm{SiO}_{2} 6.1 \%, \mathrm{Na}_{2} \mathrm{O} 24.4 \%, \mathrm{CaO} 26.9 \%$ and $\mathrm{P}_{2} \mathrm{O}_{5} 2.6 \%$ ) and all other chemicals were purchased from Guoyao Regents Company (Shanghai, China). DMEM culture medium (low-glucose and high glucose) was purchased from Gibco BRL (Rockville, MD, USA). Penicillin-streptomycin was both purchased from Invitrogen Life Technologies (Shanghai, China). Fetal bovine serum (FBS) was purchased from Hiclone (Thermo Fisher Scientific Inc., Beijing, China).

Mouse fibroblast NIH3T3 cell line was a generous gift from Prof. Hongcheng Gu of the Nano Biomedical Material Research Center, Med-X Research Institute, Shanghai Jiao Tong University. 


\subsection{Preparation of PLA/Gelatin Composite Membrane}

PLA was dissolved in a mixture of dichloromethane and hexafluoroisopropanol (mass ratio 5:2) to make a final concentration of $17.65 \%$. MNA was also dissolved in the above mixture to make a series of concentration of $2 \%, 6 \%, 12 \%$, and $24 \%$, respectively. The solution was stirred at room temperature for $4 \mathrm{~h}$ and transferred directly into a $2 \mathrm{ml}$ syringe. The electrospinning apparatus was equipped with a high-voltage statitron, a precision pump (LSP01-1A, Baoding Longer Precision Pump Co., Ltd.) and a grounded aluminum foil used as the collector. The flow rate of the polymer solution in the syringe was controlled by the precision pump at $0.05 \mathrm{~mL} / \mathrm{min}$, the voltage for electrospinning was set as $12 \mathrm{kv}$ and the tip-to-collector distance was fixed at $6 \mathrm{~cm}$.

Gelatin was dissolved in a $2.5 \%$ acetic acid solution to reach the final concentration of 7\%. BG powders (over 400 meshes) were dispersed uniformly in gelatin solution to make gelatin-BG solutions of $0.1 \%, 0.5 \%$ and $1 \%$ in concentrations. The gelatin-BG solution was casted into a Teflon mold (height $10 \mathrm{~mm}$, diameter $15 \mathrm{~mm}$ ) with the volume of $500 \mu \mathrm{l} /$ well and freeze at $-80^{\circ} \mathrm{C}$ for $2 \mathrm{hrs}$. After the $24 \mathrm{~h}$ freeze-drying process, the gelatin-BG porous scaffolds were taken out from the mold and put into 5\% glutaraldehyde-ethanol solution for $2 \mathrm{~h}$ at room temperature to allow complete cross linking. The scaffolds were preserved in ethanol for further studies.

The surface of gelatin or gelatin-BG porous scaffold was flattened by frozen slicer (CM1900UV, Leica, Germany). The PLA-MNA electrospinning membrane was cut into similar round shape with the diameter of $15 \mathrm{~mm}$. An additional 100 $\mu \mathrm{l}$ gelatin solution was added between the flattened surface of the BG scaffold and the PLA-MNA membrane to make the composite membrane. The whole composite membrane was put into 5\% glutaraldehyde-ethanol solution and cross-linked for $24 \mathrm{~h}$ at $-80^{\circ} \mathrm{C}$. Excessive double distilled water was used to wash the composite membrane to make sure there was no glutaraldehyde residue on the membrane.

\subsection{Characterization of PLA/Gelatin Composite Membrane}

The surface morphology of PLA-MNA composite electrospinning membranes and gelatin-BG porous scaffolds were observed by a scanning electron microscope (SEM, S-4800, Hitachi). The images of each sample were obtained after sputter-coating with an ultrathin layer of gold. The PLA/gelatin composite membrane was slit in the width of $1 \mathrm{~mm}$ and the longitudinal section was examined by SEM after gold coating.

\subsection{Material Degradation}

PLA and PLA-MNA composite electrospinning membranes were put in $20 \mathrm{ml}$ phosphate buffer saline (PBS) and incubated at $37^{\circ} \mathrm{C}$. The initial mass of each sample was around $15 \mathrm{mg}$ and each sample group was tested in triplicates. The duration of this experiment is $12 \mathrm{w}$. By the end of each week, the membranes 
were rinsed with plenty of water and dried for $24 \mathrm{~h}$ using freeze-drying method. The PBS in the rest of the groups was changed into fresh one.

\subsection{MNA Releasing}

Composite membranes with different content of MNA were weighed and cut into $0.1 \mathrm{~g}$ in mass. The membranes were immersed into $20 \mathrm{ml}$ PBS individually and incubated at $37^{\circ} \mathrm{C}$. One $\mathrm{ml}$ supernatant was removed for analysis at different time points, from $3 \mathrm{~h}$ to $800 \mathrm{~h}$. After each sample removal, $1 \mathrm{ml}$ fresh PBS was added. MNA concentration was determined its OD value at $320 \mathrm{~nm}$ using a UV spectrophotometer (Bio TEK Synergy 2)

\subsection{Biocompatibility and Cell Proliferation}

NIH3T3 cells were cultured in DMEM high glucose culture medium supplemented with $10 \%$ fetal bovine serum (FBS, Hiclone, Thermo Fisher Scientific Inc., Beijing, China) and $1 \%$ penicillin-streptomycin (Invitrogen, USA). The cells were incubated in a $5 \% \mathrm{CO}_{2}$ atmosphere at $37^{\circ} \mathrm{C}$. The samples were sterilized by $75 \%$ ethanol. Titanium rings were used to immobilize the membranes to prevent the membrane from floating during the experiment. NIH3T3 were seeded on the membrane with the density of $2 \times 10^{4} /$ well in a 12 -well plate.

Live-dead cell staining (LIVE/DEAD Viability/Cytotoxicity Kit, Molecular Probes Inc., USA) was applied to evaluate the biocompatibility of the sample. After NIH 3T3 cells being cultured for 3 and 7 days, the membranes with the cells were removed from the culture medium and washed twice with DPBS (Dulbecco's phosphate-buffered saline). Calcein $\mathrm{AM}$ and EthD-1 were diluted to $2 \mu \mathrm{M}$ and 4 $\mu \mathrm{M}$ by $\mathrm{D}$-PBS for staining, and the reaction was incubated in $37^{\circ} \mathrm{C}$ for 15 mins according to the instruction. The live ones were visualized under $485 \mathrm{~nm}$ exciting wavelength under a fluorescence microscopy (DMI3000B, Leica, Germany), while the dead cells were visualized under $530 \mathrm{~nm}$ exciting wavelength. The fluorescence microscope images were merged by Image $\mathrm{J}$.

Cell proliferation was determined by CCK- 8 assay (Beyotime Co., China). NIH 3 T3 were seeded on the membrane with the density of $2 \times 10^{4} /$ well, and the cells cultured on the cell culture plate with the same density were served as control. At day 1, 4 and 7, the relative cell numbers was determined by CCK- 8 according to the supplier's procedure.

\subsection{In Vitro Bioactivity of Gelatin-BG Porous Scaffold}

Simulated body fluid (SBF) was prepared according to the protocol described by Kokubo et al. The gelatin and gelatin-BG porous scaffolds of different content of BG were soaked in $\mathrm{SBF}$ at $37^{\circ} \mathrm{C}$ and $\mathrm{pH} 7.4$ for 7 days, and the SBF was refreshed every other day. All the scaffolds were removed from the solution and washed abundantly with double-deionized water at the end of the experiment. Freeze-drying method was used to prepare the scaffold for further morphology analysis and composition examination. 
The surface morphology was observed by SEM. FTIR spectroscopy was applied to examine the formation of hydroxyapatite (HA) on each sample after the immersion in SBF. The samples were crushed and then mixed with potassium bromide in 1:100 proportions. The mixture was compressed to a $1 \mathrm{~mm}$ slice under the pressure of $10 \mathrm{Mpa}$ before the FTIR detection. The FTIR spectra of the wavelength range $4000-400 \mathrm{~cm}^{-1}$ were recorded by FTIR spectrometer (Nicolet Co., USA).

\subsection{Ions Release of the PLA/Gelatin Composite Membrane}

After being freeze-dried, $0.1 \mathrm{~g}$ gelatin-BG porous scaffolds with different content of BG were soaked in $20 \mathrm{~mL}$ PBS individually and incubated at $37^{\circ} \mathrm{C}$. At each designated time points $(6 \mathrm{~h}, 12 \mathrm{~h}, 24 \mathrm{~h}, 2 \mathrm{~d}, 3 \mathrm{~d}, 4 \mathrm{~d}, 5 \mathrm{~d}, 6 \mathrm{~d}, 8 \mathrm{~d}, 10 \mathrm{~d}, 12 \mathrm{~d}, 14 \mathrm{~d}$, $16 \mathrm{~d}, 20 \mathrm{~d}, 24 \mathrm{~d}, 28 \mathrm{~d}$ and $32 \mathrm{~d}$ ), respectively, $1.5 \mathrm{~mL}$ supernatant was removed from each sample and $1.5 \mathrm{~mL}$ fresh PBS was returned into each sample to compensate the volume loss. All the samples were stored at $4^{\circ} \mathrm{C}$ before the analysis. To determine the elemental concentrations of calcium and silicon ( $\mathrm{Ca}$ and $\mathrm{Si}$ ), inductively coupled plasma optical emission spectroscopy (ICPOES-Varian 715-ES, USA) was used to analyze the samples.

\subsection{Osteogenic Differentiation of rBMSCs Cultured on PLA/Gelatin Composite Membrane}

The rBMSCs were isolated from the bone marrow of 4 week-age DW rat. For consistency, the third generation of the cells was used. Seeding density of rBMSCs was $1 \times 10^{5}$ cells per well in a 6-well plate. Total RNA was isolated by TRIZOL Reagent (Invitrogen, US) after 3, 5 and 7 days of cell culture according to the manufacturer's instructions. RNA yields were determined by spectrophotometry (Nanodrop, Thermo Fisher Scientific) at $260 \mathrm{~nm}$. Complementary DNA (cDNA) was synthesized using reverse transcription (RT)-PCR, which was performed by Rever Tra Ace- $\alpha$ Transcriptase Kit (Toyobo Osaka, Japan).

Quantitative Real-Time PCR (qRT-PCR) was conducted using the SYBR Green Real-time PCR Master Mix Kit (Toyobo, Osaka, Japan) according to the manufacturer's protocol. cDNA was used as template and glyceraldehyde-3-phosphate dehydrogenase (GAPDH) was considered as an internal control to determine the relative quantification of target gene expression. The primers were purchased from Sangon Biotech (Shanghai, China) and primer sequences are:

BSP-Forword5'-ACAAATGCTTCCGTTTGTAGCGGC-3';

BSP-Reverse5'-ACAGCCCTGATTTACGATGACCCT-3';

ALP-Forward5'-AATCGGAACAACCTGACTGACCCT-3';

ALP-Reverse5'-AATCCTGCCTCCTTCCACTAGCAA-3';

GAPDH-Forward5'-ACAAGATGGTGAAGGTCGGTGTGA-3';

GAPDH-Reverse5'-AGCTTCCCATTCTCAGCCTTGACT-3'.

qRT-PCR was carried out in a $10 \mu \mathrm{L}$ reaction mixture and performed on the 7900 HT Fast Real-Time PCR system (Applied Biosystems, Singapore) by using the following thermal profile: $1 \mathrm{~min}$ at $95^{\circ} \mathrm{C}$, followed by 45 cycles of $15 \mathrm{~s}$ at 
$95^{\circ} \mathrm{C}, 15 \mathrm{~s}$ at $60^{\circ} \mathrm{C}$, and $45 \mathrm{~s}$ at $72^{\circ} \mathrm{C}$. The dissociation stage $\left(95^{\circ} \mathrm{C}\right.$ for $15 \mathrm{~s}, 60^{\circ} \mathrm{C}$ for $15 \mathrm{~s}$ ) was also included after PCR amplification to make sure only one sharp peak is detected at the melting temperature of amplicon. qRT-PCR data were analyzed by the comparative CT method. To investigate the cell osteogenic differentiation of gelatin-BG porous scaffold, levels of mRNA of the osteogenic cytokines alkaline phosphatase (Alpl) and bone sialoprotein (Bsp) were determined by qRT-PCR.

\subsection{Statistical Analysis}

The values were expressed as means (standard deviation, SD). Whenever appropriate, two-tailed Student's $t$-test was used to discern the statistical difference between groups. A probability value $(\mathrm{P})$ of less than 0.05 was considered to be statistically significant.

\section{Results}

\subsection{Characterization of PLA/Gelatin Composite Membrane}

PLA-MNA/gelatin-BG composite membrane was prepared by the procedures described above. The composite membrane showed an asymmetric structure (Figure 1(a) and Figure 1(b)). One side was the PLA electrospun membrane and the other side was the porous gelatin scaffold. The PLA or PLA-MNA electrospun fibers were uniform in diameters, except that the surface roughness of the fibers increased while the content of MNA in PLA-MNA increasing, as shown in Figures $1(\mathrm{c})-(\mathrm{g})$. The average pore size of the PLA membrane is $3.18 \pm 0.57 \mu \mathrm{m}$. The average thickness of the membrane is about 50 to $100 \mu \mathrm{m}$. The con-focal microscopic image (Figure $1(\mathrm{~h})$ ) demonstrated a sponge-like structure of gelatin scaffold. The 3D reconstruction from the con-focal images showed that the pores are interconnected in the gelatin matrix, indicating the suitability for $3 \mathrm{D}$ cell culture in the future. Small particles were presented on the surface of scaffolds with the existence of different percentage of BG when making gelatin scaffolds (Figures 1(i)-(1)), which demonstrating BG nanoparticles were uniformly embedded in gelatin scaffold. The average thickness of the gelatin scaffold was about $0.85 \mathrm{~mm}$, which contributed most of the overall thickness of the GTR membrane (Figure 1(b)).

It is very important to mention two distinguished attributes of the GTR membrane. When implanted, the PLA side faces the periodontal soft tissue. Since the pore size of the PLA elestrospun membrane is too small to allow any cell to penetrate, it works as a cell barrier for nutrition and waste exchange only. This is extremely important since the biggest obstacle of the regeneration of the soft tissue is the invasion of the fibroblast cells which grows much faster than the periodontal cells. On the other hand, the 3D structure of the gelatin scaffold provides a very suitable environment for the regeneration of the alveolar bone. Meanwhile, it increased significantly the tensile strength of the GTR membrane compared to the pure PLA membrane, which provides enough mechanical support to match the regeneration rate of both the hard and the soft tissue. 

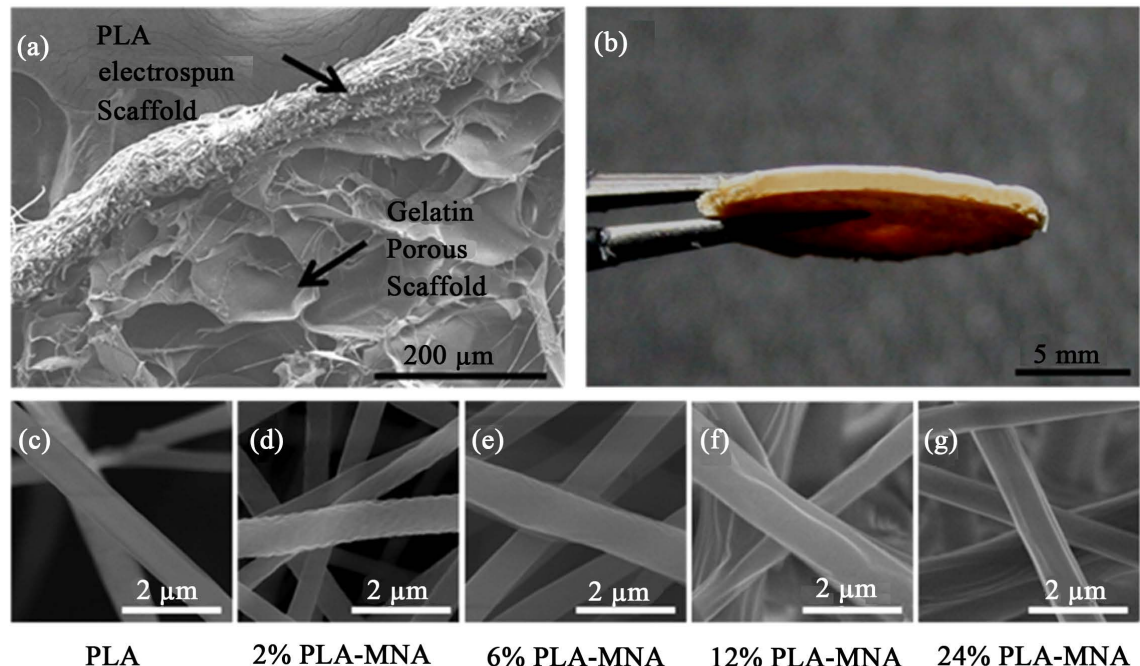

PLA

2\% PLA-MNA

$\%$ PLA-MNA

12\% PLA-MNA

24\% PLA-MNA

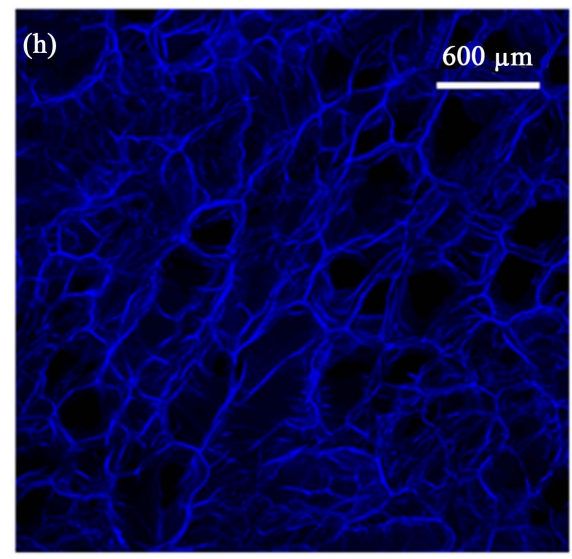

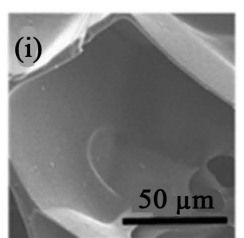

Gelatin

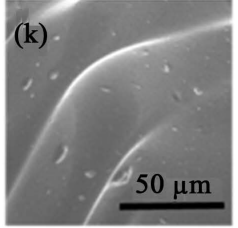

$0.5 \%$ gelatin-BG

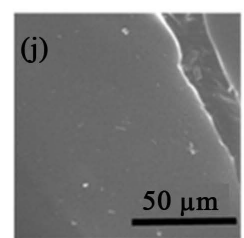

$0.1 \%$ gelatin-BG

(1)

$50 \mu \mathrm{m}$

$1 \%$ gelatin-BG

Figure 1. Characterization of PLA-MNA/gelatin-BG composite membrane. SEM image (a) and digital photo (b) of PLA/gelatin composite membrane; (c) SEM images of PLA eletrospun fiber; ((d)-(g)) PLA-MNA eletrospun fibers with different content of MNA; (h) Confocal image of gelatin porous scaffold; ((i)-(l)) SEM images of gelatin porous scaffolds with different content of BG.

\subsection{MNA Releasing Profile}

Before mixing MNA with PLLA for electrospinning, we first tested the cytotoxicity of MNA on NIH3T3 cells in order to determine the safe concentration of MNA in use. NIH3T3 cells were selected as an in vitro model cell to test cytotoxicity. According to the OD value of the cultured cells, the treatment concentration below $70 \mathrm{mg} / \mathrm{ml}$ showed no sign of cytotoxicity, which means as long as the releasing concentration from PLLA is less than or equal to $70 \mathrm{mg} / \mathrm{ml}, \mathrm{MNA}$ has no toxic effect on the surrounding cells (Figure 2(a)).

Following the above experiment, MNA was mixed with PLA solution with the proportion range from $2 \%$ to $24 \%$. The MNA releasing profiles was determined during an $800 \mathrm{~h}$ period. According to the result (Figure 2(b)), MNA showed a burst release in the first four-day period at higher composition of $12 \%$ and $24 \%$. However, the releasing curve is much smoother when the composition of MNA was decreased to $2 \%$. Since the concentration of MNA was very stable, keeping 


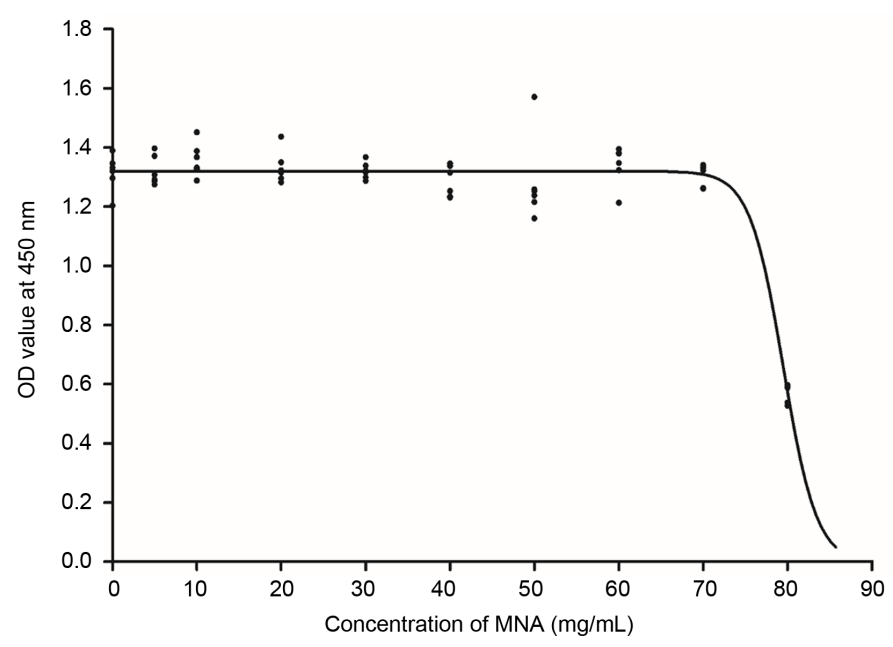

(a)

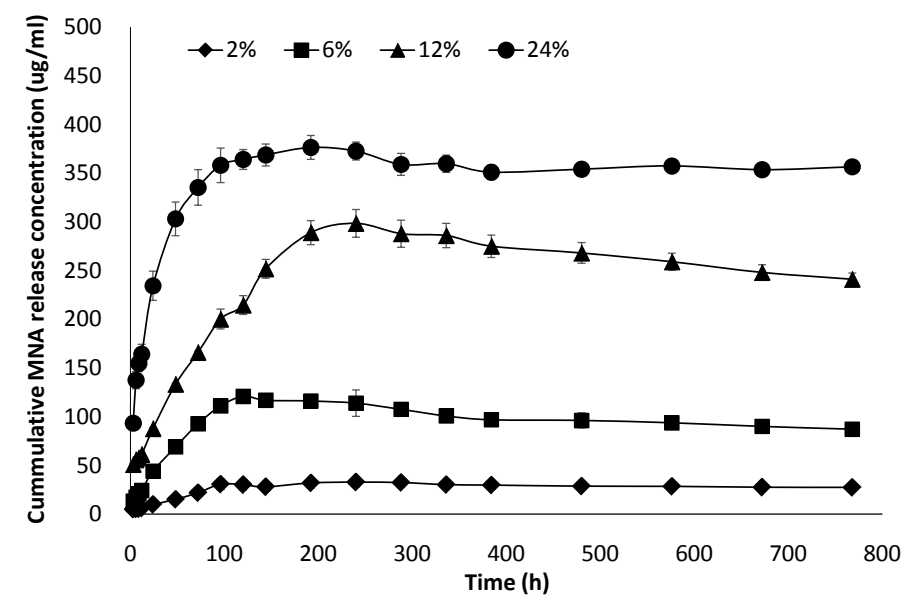

(b)

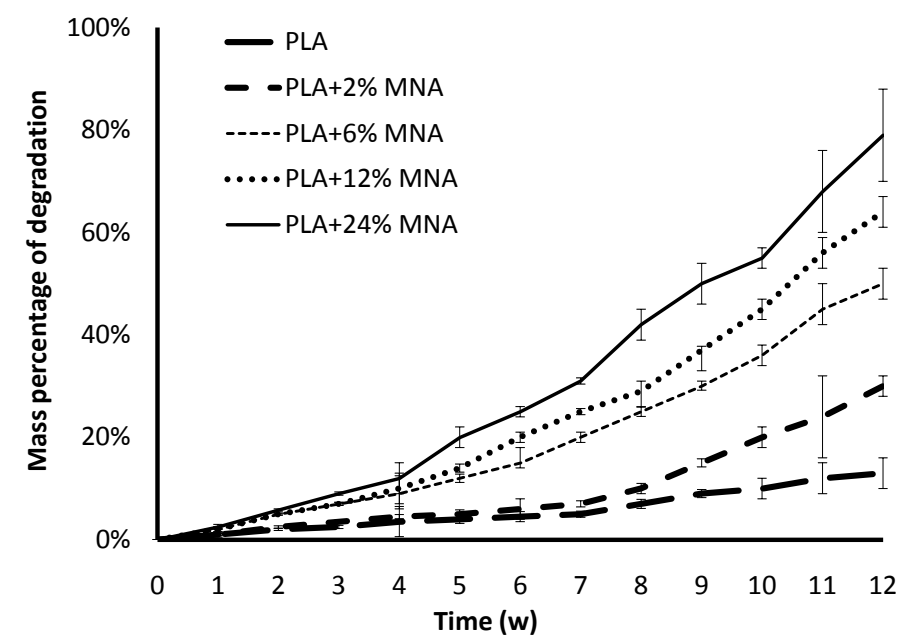

(c)

Figure 2. The metronidazole releasing profile. (a) The inhibition effect of different MNA concentration on NIH3T3 cell's proliferation; (b) The MNA releasing curve from PLA-MNA electrospun membranes with different MNA compositions; (c) The mass percentage of degradation of PLA-MNA electrospun membranes with different MNA compositions. 
with the baseline of about $20 \mu \mathrm{g} / \mathrm{ml}$, which was well below the toxicity concentration of MNA (70 mg/ml). Therefore, MNA compositions from $2 \%$ to $24 \%$ are all acceptable concentrations in fabricating of the PLA electrospun membrane.

Moreover, the degradation of the PLA/gelatin composite membrane over up to 3 months was evaluated. According to our results, the degradation percentage increased with the increasing of MNA composition (Figure 2(c)). The membrane became more and more fragile if there were higher concentration of MNA and the tensile strength of the membrane decreased very quickly, especially at the late stage of the degradation. In order to maintain enough tensile strength of the GTR membrane for the cells to attach since the recovery of the ligament was at least 3 months, we decided that $6 \%$ MNA was the most suitable for the membrane with its degradation percentage less than $50 \%$ by the end of 3 months.

\subsection{In Vitro Bioactivity of Gelatin-BG Porous Scaffold}

In this design, BG was selected to reinforce the mechanical strength of the GTR membrane. To functionalize the gelatin scaffold, BG was considered to be combined with the scaffold. SEM image confirmed that BG powders were embedded evenly both inside and outside the gelatin scaffold. Since embedding BG in the gelatin may affect its bioactivity, pure gelatin and gelatin-BG scaffolds with different content of BG were soaked in SBF to examine whether there was the formation of HA on the surface. Figures 3(a)-(d) showed the SEM images of the samples after the immersion. The surface of pure gelatin scaffold remained smooth while some deposits were formed on gelatin-BG composite scaffolds. The morphology of the deposits was showed in the high-magnification images, which was similar with the typical worm-like morphology of HA. FITR patterns of the samples were recorded to identify the component of deposition. The characteristic double peaks of $\mathrm{HA}$ at 601.16 and $561.48 \mathrm{~cm}^{-1}$ were observed after the immersion in SBF for gelatin-BG composite scaffolds (Figure 3(e)).

Therefore, it can be concluded that there were HA formation on the surface of gelatin-BG composite scaffolds while in biological environment.
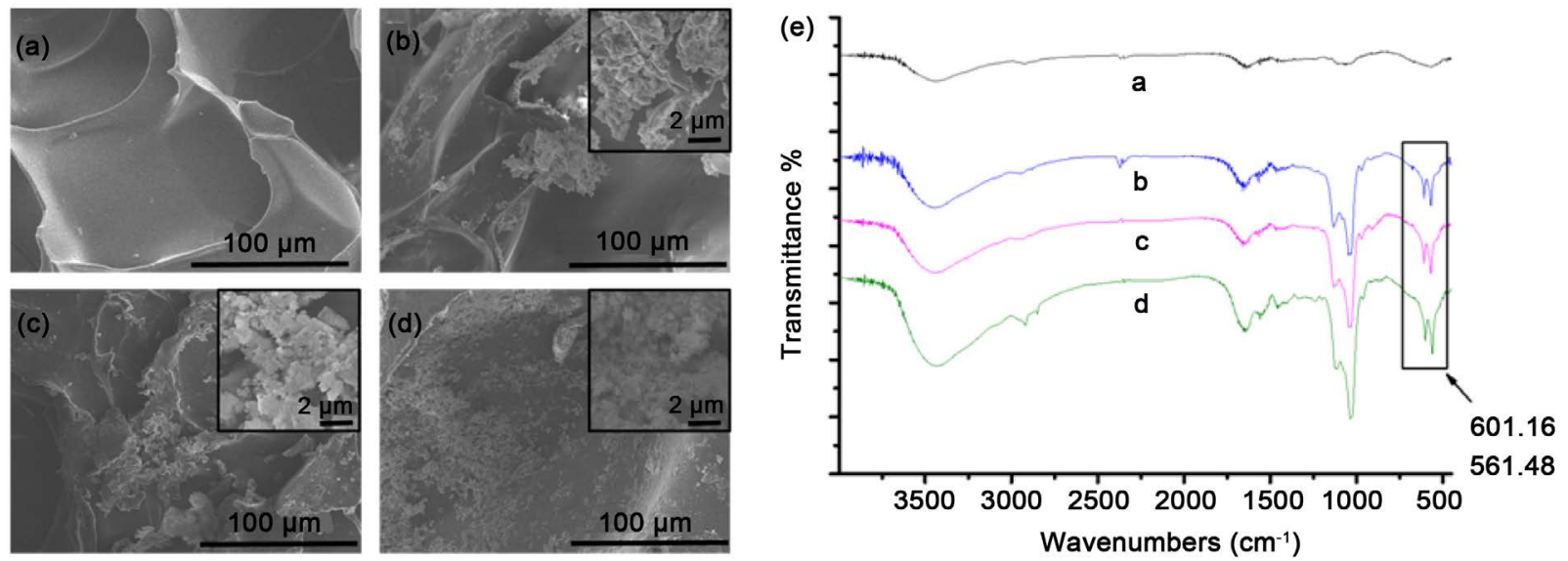

Figure 3. Characterization of gelatin-BG porous scaffold. SEM images ((a)-(d)) and FTIR spectra (e) of gelatin-BG composite scaffolds after soaking in SBF for 7 days. (a) gelatin; (b) $0.1 \%$ gelatin-BG; (c) $0.5 \%$ gelatin-BG; (d) $0.5 \%$ gelatin-BG. 


\subsection{Biocompatibility of PLA/Gelatin Composite Membrane}

The biocompatibility of the composite membrane was tested by culturing NIH3T3 cells and expressed by the percentage of living cells compared to the control. Healthy NIH3T3 cells are in spindle-shape morphology but the unhealthy ones could not hold the normal shape and became round-shaped and even detached from the membrane surface. Figure 4(a) showed the result of 3-day and 7-day culture on the PLA-MNA side of the composite membrane. The results showed that the proliferation rate of the cells attached to pure PLA was about $90 \%$ while that of the cells attached to PLA-MNA membrane after 3-day's culture is about 98\%. After 7-day's culture, the cell density on pure PLA membrane increased, but still slightly lower than that on the PLA-MNA membrane. Meanwhile, the cell proliferation result of NIH3T3 after being cultured on PLA-MNA membranes for 1, 4 and 7 days was shown in Figure 4(o). During the whole culture
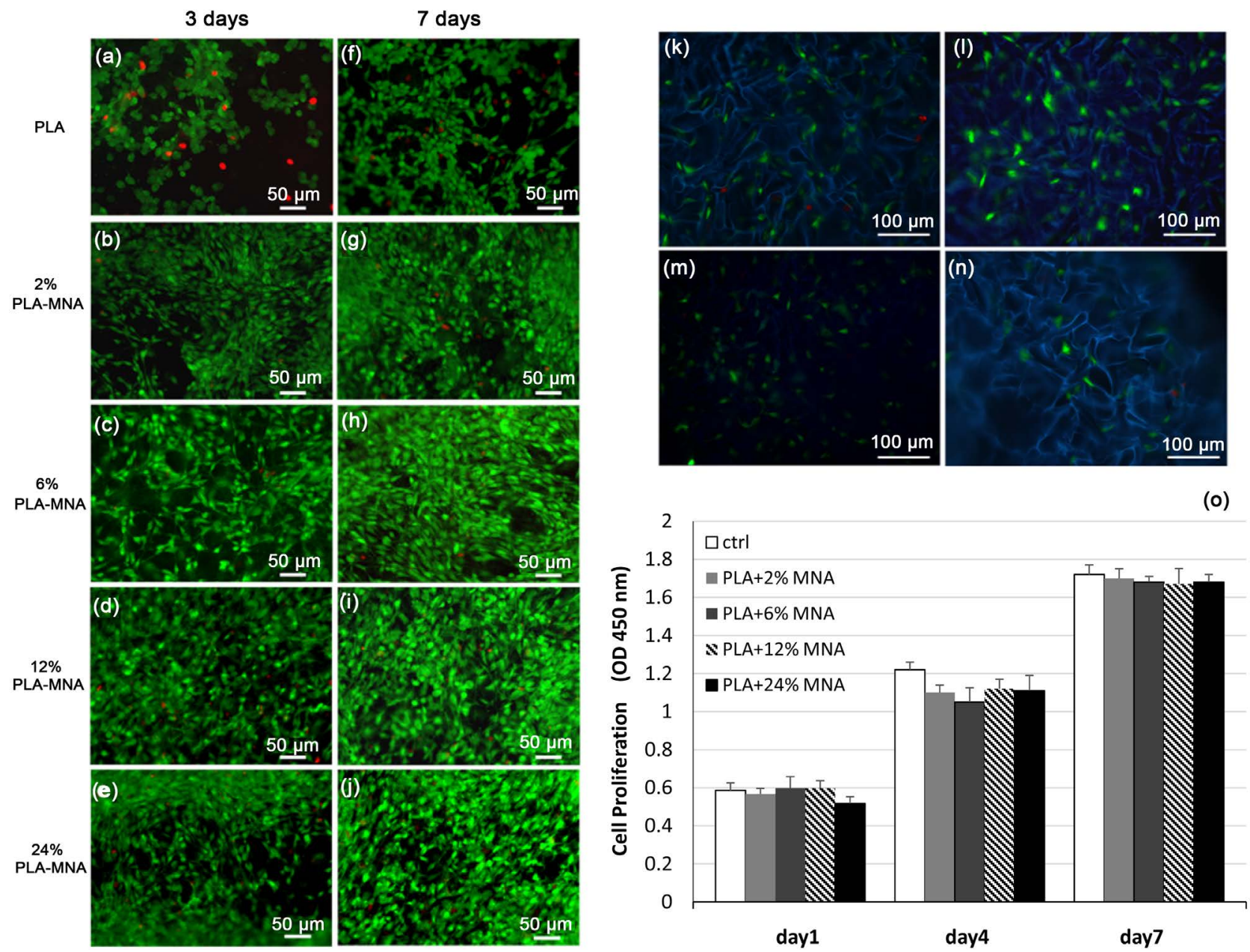

Figure 4. Biocompatibility and cell proliferation on both of the PLA-MNA membrane side and the Gelatin-BG side of the composite membrane. ((a)-(j)) Fluorescence microscope images of NIH3T3 cells after Live-dead staining. NIH3T3 cells were cultured on PLA ((a), (f)) and PLA-MNA ((b)-(e), (g)-(j)) membranes for 3 days ((a)-(e)) and 5 days ((f)-(j)); (o) Quantitation of NIH3T3 proliferation on PLA-MNA membrane on day 1, 4 and 7; ((k)-(n)) Fluorescence microscope images of rBMSCs cultured for 5 days on gelatin-BG composite scaffolds after Live-Dead staining; (k) Gelatin; (l) $0.1 \%$ gelatin-BG; (m) 0.5\% gelatin-BG; (n) $0.5 \%$ gelatin-BG. 
period, the OD values increased with no significant difference $(P>0.05)$ observed between PLA-MNA with different content of MNA and the control at the same culture period. In addition, Figures $4(\mathrm{k})$-(n) showed the result of 5-day culture of rBMSCs on the gelatin side of the composite membrane. The results were similar to the other side of the membrane that rBMSCs showed no significant difference between the control and the gelatin with different contents of BG. Taken together, these results demonstrated that the existence of both MNA and BG in the PLA-gelatin composite membrane did not affect the proliferation of NIH3T3 cells, meaning the biocompatibility of the PLA/gelatin composite membrane was satisfactory.

\section{5. $\mathrm{Ca}^{2+}$ and $\mathrm{SiO}_{4}^{2-}$ Ions Releasing from the PLA/Gelatin Composite Membrane}

The ion releasing profiles were shown in Figure 5(a) and Figure 5(b). The releasing profiles for $\mathrm{Ca}^{2+}$ and $\mathrm{SiO}_{4}^{2-}$ were similar. A burst releasing period was observed in the first $24 \mathrm{~h}$ and both ions reached a peak maintained a smooth releasing afterwards. The equilibrium concentration was determined by the composition of BG in gelation. The higher the composition, the higher the equilibrium concentration.

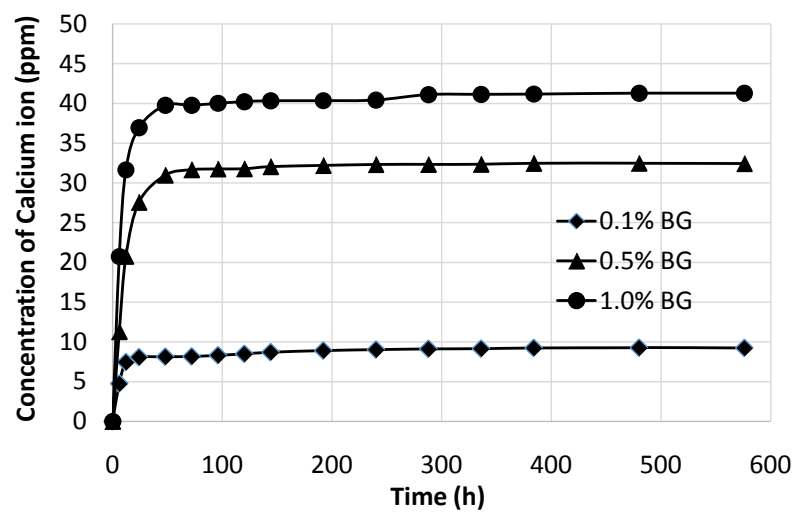

(a)

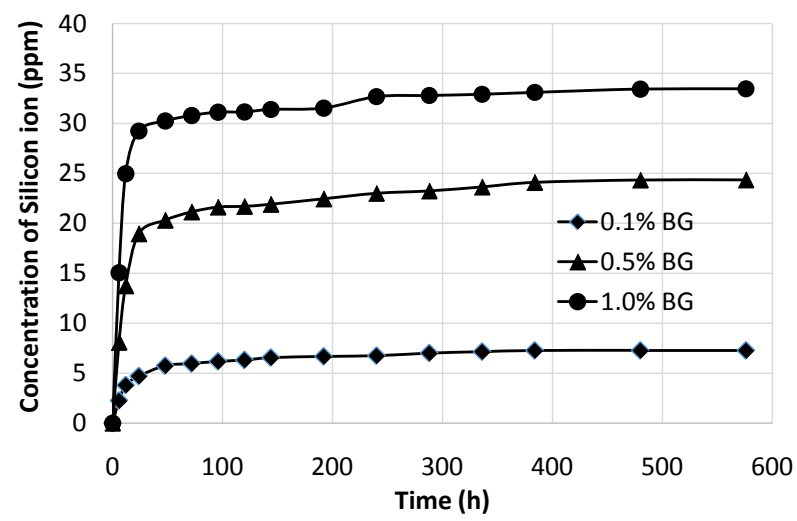

(b)

Figure 5. Cumulative release curve in PBS of $\mathrm{Ca}(\mathrm{a})$ and $\mathrm{Si}$ (b) from PLA/gelatin scaffolds in 32 days. 


\subsection{Osteogenic Differentiation of rBMSCs Cultured on the PLA/Gelatin Composite Membrane}

To determine whether there would be any functional alteration after integrated into gelatin scaffold, the induction of BG for osteogenesis was evaluated using rat derived bone mesenchymal stem cells (rBMSC). Figure 6 showed the expression levels of two genes, alkaline phosphatase (Alpl) and bone cialoprotein (Bsp). Since Alp is expressed in the new bone and Bsp is expressed in the newly synthesized dentinum, both can be considered as regeneration markers. It has been demonstrated that pure gelatin has no induction of Alpl and Bsp gene expression in BMSC but when BG was introduced to the composite scaffold, both Alp and Bsp mRNA level was induced significantly $(P<0.001)$. Alpl was upregulated to about 3 folds over the control and Bsp was upregulated over 4 folds compared to the control. The results suggested that $B G$ maintained its function in the composite scaffold. In combined with the result in Figure 5, the $\mathrm{Ca}^{2+}$ and $\mathrm{SiO}_{4}^{2-}$ concentrations were between $10 \mathrm{ppm}$ to $40 \mathrm{ppm}$. The results showed that trace ion concentration $(10-40 \mathrm{ppm})$ can already direct the rBMSCs differentiate towards osteogenesis.

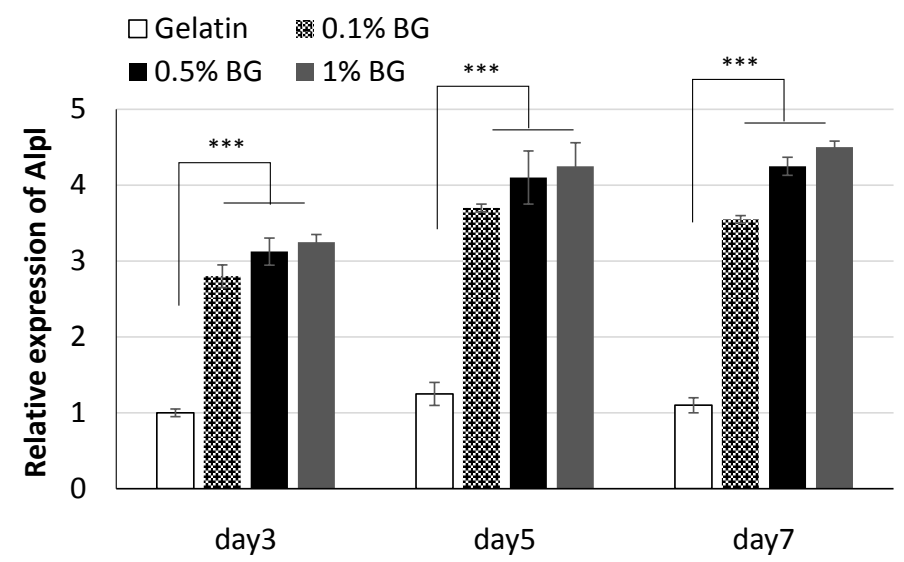

(a)

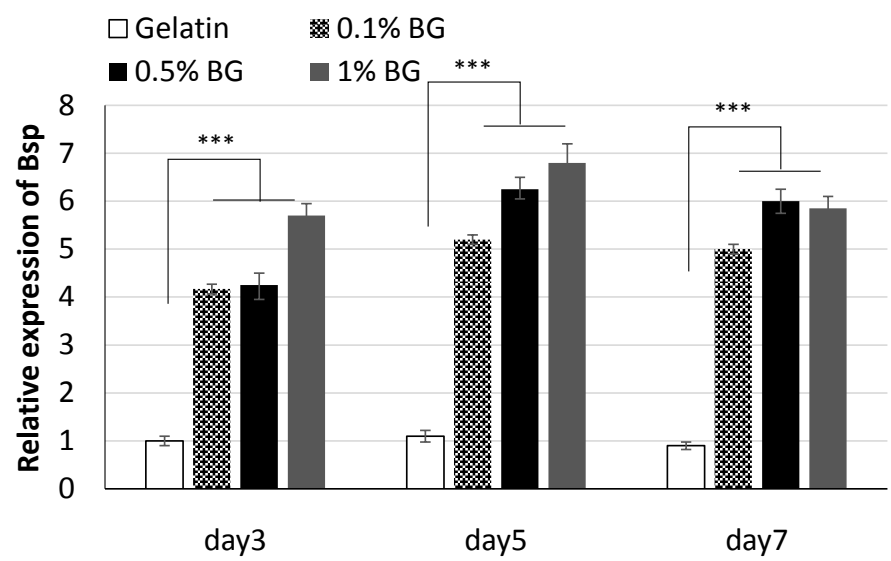

(b)

Figure 6. Alpl (a) and Bsp (b) expression of rBMSCs cultured on gelatin-BG composite scaffolds after 3,5 and 7 days. $(* * P<0.001)$. 


\section{Discussion}

Development of new cementum with PDL fibers connected to alveolar bone is the main goal of periodontal regeneration [11] [12]. Based on different clinical conditions, both non-degradable and bio-degradable GTR membranes are used in dental clinics. Expanded polytetrafluoroethylene membrane (e-PTFE) and its derivatives are the most common non-degradable GTR membrane. Commercialized bio-degradable GTR membranes include Vicryl ${ }^{\circledast}$, Guidor ${ }^{\circledast}$, Epi-Guide ${ }^{\circledast}$, and Resolut $^{\oplus}$, most of which are PLA-based polymericmaterial. In our previous study, a PLA/chitosan membrane has been successfully fabricated and showed great potential to avoid fibroblast invasion which was an unfavorable side effect in periodontal regeneration [7]. Base on that research, we continue to improve the design to make it more suitable for the regeneration of both alveolar bone and gingiva tissue.

The highlight of this new GTR is the "reverse" design that to create an unsymmetrical bi-layer GTR membrane, inspired by biology and anatomy. In order to meet the different requirements for regeneration from alveolar bone and the gingiva epithelial tissue, PLA and gelatin were selected as the major composition of the scaffold. Bottino et al. [6] presented a smartly designed functionally graded structure using PLA electrospinning membrane sandwich to tailor the degradation time and proper tensile strength. However, their "sandwich" design was still symmetric. Both gelatin and PLA can be fabricated to mimic the natural extracellular matrix and showed a very promising advantage in tissue regeneration [13]. The problem of PLA is its degradation rate is relatively low. To solve these problems, MNA was mixed with PLA solution for electrospinning. This step is quite critical because the addition of MNA makes it easier to degrade in physiological condition and the degradation speed is fine-tunable by change the amount of MNA. Clinical experience has reflected that bacterial infection is not only involved in the etiology of periodontitis but also becomes an obstacle for tissue regeneration [14]. MNA is an effective drug for treating anaerobic bacteria infection in mouth and intestinal tract. The suggested dosage for an adult is $1.2 \mathrm{~g}$ to $1.8 \mathrm{~g}$ per day. Since the degradation time of PLA needs to be adjustable to match the regeneration time of the ligament for at least 3 months, PLA was to mix with MNA in the electrospinning solution to optimize the PLA degradation rate. In combined with the idea that enough tensile strength of the GTR membrane has to be maintained, we finally decided that 6\% MNA in PLA composition was the most suitable for the membrane with its degradation percentage less than $50 \%$ by the end of 3 months.

Gelatin, on the other hand, increased the tensile strength of the whole GTR during regeneration, which is also a preferable feature needs to be mentioned since the addition of MNA increased the degradation rate but meanwhile decrease the tensile strength need for tissue regeneration. BG is a traditional biomaterial which has been safely applied in dentistry for a long time. It has been reported that the ions released from BG could in certain extent form a favorable 
microenvironment for the differentiation of the BMSCs [15]. BG can form a carbonated apatite layer between hard and soft tissues. Bioactive glass ceramics have been reported to influence osteoblastic cell differentiation with an increase in the level of differentiation markers like alkaline phosphatase (ALP), osteocalcin and osteopontin [16]. In this case, although the overall ion releasing profile is slightly different from that of BG without embedding in the gelatin gel, the $\mathrm{Ca}^{2+}$ and $\mathrm{SiO}^{4+}$ ions were still released from the gel and they can reach the induction concentration to up-regulate the expression of Alpl and Bsp, indicating directed osteogenesis change of the BMSCs.

It should be noted that some of the patients need GTR membrane implant usually have other clinical complications such as diabetes, blood diseases, and recent cardio-vascular outbreaks. In those patients, the healing progress is usually slower. Therefore, future research emphasis need to be laid on more complicated in vivo study.

\section{Conclusion}

In conclusion, although simple and cheap materials were used, the optimized combination and bio-inspired design endow our new GTR membrane great clinical application potential in treating periodontitis.

\section{Acknowledgements}

The authors want to thank Prof. Song Li of University of California Los Angeles for his critical revision of this manuscript. The work presented is supported by the Science and Technology Commission of Shanghai Municipality (16140901900).

\section{References}

[1] Pihlstrom, B.L., Michalowicz, B.S. and Johnson, N.W. (2005) Periodontal Diseases. The Lancet, 366, 1809. https://doi.org/10.1016/S0140-6736(05)67728-8

[2] Longo, P.L., Artese, H.P., Rabelo, M.S., Kawamoto, D., Foz, A.M., Romito, G.A., Dib, S.A. and Mayer, M.P. (2014) Serum Leveis of Inflammatory Markers in Type 2 Diabetes Patients with Chronic Periodontitis. Journal of Applied Oral Science, 22, 103-108. https://doi.org/10.1016/S0140-6736(05)67728-8

[3] Masi, S., Gkranias, N., Li, K., Salpea, K.D., Parkar, M., Orlandi, M., Suvan, J.E., Eng, H.L., Taddei, S., Patel, K., Darbar, U., Donos, N., Deanfield, J.E., Hurel, S., Humphries, S.E. and D'Aiuto, F. (2014) Association between Short Leukocyte Telomere Length, Endotoxemia, and Severe Periodontitis in People with Diabetes: A Cross-Sectional Survey. Diabetes Care, 37, 1140-1147. https://doi.org/10.1016/S0140-6736(05)67728-8

[4] Costa, T.H., de Figueiredo Neto, J.A., de Oliveira, A.E., Lopes e Maia Mde, F. and de Almeida, A.L. (2014) Association between Chronic Apical Periodontitis and Coronary Artery Disease. Journal of Endodontics, 40, 164-165. https://doi.org/10.1016/j.joen.2013.10.026

[5] Bottinoa, M.C., Thomasb, V., Schmidtc, G., Vohrab, Y.K., Chua, T.-M.G., Kowolikd, M.J. and Janowski, G.M. (2012) Recent Advances in the Development of GTR/GBR Membranes for Periodontal Regeneration-A Materials Perspective. Dental Materials, 28, 703-721. https://doi.org/10.1016/j.dental.2012.04.022 
[6] Bottino, M.C. and Thomas, V. (2015) Membranes for Periodontal Regeneration-A Materials Perspective. Frontiers of Oral Biology, 17, 90-100.

[7] Chen, S., Hao, Y.T., Cui, W.G., Chang, J. and Zhou, Y. (2013) Biodegradable Electrospun PLLA/Chitosan Membrane as Guided Tissue Regeneration Membrane for Treating Periodontitis. Journal of Materials Science, 48, 6567-6577. https://doi.org/10.1007/s10853-013-7453-z

[8] Srinivasan, S., Jayasree, R., Chennazhi, K.P., Nair, S.V. and Jayakumar, R. (2012) Biocompatible Alginate/Nano Bioactive Glass Ceramic Composite Scaffolds for Periodontal Tissue Regeneration. Carbohydrate Polymers, 87, 274-283. https://doi.org/10.1016/j.carbpol.2011.07.058

[9] Bottino, M., Thomas, V. and Janowski, G. (2011) A Novel Spatially Designed and Functionally Graded Electrospun Membrane for Periodontal Regeneration. Acta Biomaterialia, 7, 216-224. https://doi.org/10.1016/j.actbio.2010.08.019

[10] Liu, X., Smith, L.A., Hu, J. and Ma, P.X. (2009) Biomimetic Nanofibrous Gelatin/Apatite Composite Scaffolds for Bone Tissue Engineering. Biomaterials, 30, 2252-2258. https://doi.org/10.1016/j.biomaterials.2008.12.068

[11] Kinane, D.F., Stathopoulou, P.G. and Papapanou, P.N. (2017) Periodontal Diseases. Nature Reviews Disease Primers, 3, 17038. https://doi.org/10.1038/nrdp.2017.38

[12] Sheikh, Z., Hamdan, N., Ikeda, Y., Grynpas, M., Ganss, B. and Glogauer, M. (2017) Natural Graft Tissues and Synthetic Biomaterials for Periodontal and Alveolar Bone Reconstructive Applications: A Review. Biomaterials Research, 21, 9. https://doi.org/10.1186/s40824-017-0095-5

[13] Negahi Shirazi, A., Fathi, A., Suarez, F.G., Wang, Y., Maitz, P.K. and Dehghani, F. (2016) A Novel Strategy for Softening Gelatin-Bioactive-Glass Hybrids. ACS Applied Materials \& Interfaces, 8, 1676-1686. https://doi.org/10.1021/acsami.5b09006

[14] Lee, B.S., Lee, C.C., Wang, Y.P., Chen, H.J., Lai, C.H., Hsieh, W.L. and Chen, Y.W. (2016) Controlled-Release of Tetracycline and Lovastatin by Poly (D,L-Lactide-co-Glycolide Acid)-Chitosan Nanoparticles Enhances Periodontal Regeneration in Dogs. International Journal of Nanomedicine, 11, 285-297.

[15] Zhou, Y., Li, H., Lin, K., Zhai, W., Gu, W. and Chang, J. (2012) Effect of Heat Treatment on the Properties of $\mathrm{SiO}_{2}-\mathrm{CaO}-\mathrm{MgO}-\mathrm{P}_{2} \mathrm{O}_{5}$ Bioactive Glasses. Journal of Materials Science: Materials in Medicine, 23, 2101-2108. https://doi.org/10.1007/s10856-012-4699-y

[16] Xie, X.H., Yu, X.W., Zeng, S.X., Du, R.L., Hu, Y.H., Yuan, Z., Lu, E.Y., Dai, K.R. and Tang, T.T. (2010) Enhanced Osteointegration of Orthopaedic Implant Gradient Coating Composed of Bioactive Glass and Nanohydroxyapatite. Journal of Materials Science: Materials in Medicine, 21, 2165-2173.

https://doi.org/10.1007/s10856-010-4077-6 